\title{
Age-determined expression of priming protease TMPRSS2 and localization of SARS-CoV-2 in lung epithelium
}

Bryce A. Schuler, ${ }^{1}$ A. Christian Habermann, ${ }^{2}$ Erin J. Plosa, ${ }^{1}$ Chase J. Taylor, ${ }^{2}$ Christopher Jetter, ${ }^{1}$ Nicholas M. Negretti, Meghan E. Kapp, ${ }^{3}$ John T. Benjamin, ${ }^{1}$ Peter Gulleman, ${ }^{1}$ David S. Nichols, ${ }^{2}$ Lior Z. Braunstein, ${ }^{4}$ Alice Hackett, ${ }^{1}$ Michael Koval, ${ }^{5}$ Susan H. Guttentag, ${ }^{1}$ Timothy S. Blackwell, ${ }^{2,6,7}$ Steven A. Webber, ${ }^{1}$ Nicholas E. Banovich, ${ }^{8}$ Vanderbilt COVID-19 Consortium Cohort, ${ }^{9}$ Human Cell Atlas Biological Network, ${ }^{10}$ Jonathan A. Kropski, ${ }^{2,6,7}$ and Jennifer M.S. Sucre ${ }^{1,6}$

'Department of Pediatrics, 'DDivision of Allergy, Pulmonary and Critical Care Medicine, Department of Medicine, and ${ }^{3}$ Department of Pathology, Microbiology, and Immunology, Vanderbilt University Medical Center, Nashville, Tennessee, USA. ${ }^{4}$ Department of Radiation Oncology, Memorial Sloan Kettering Cancer Center, New York, New York, USA. ${ }^{5}$ Division of Pulmonary, Allergy, Critical Care, and Sleep Medicine, Emory University, Atlanta, Georgia, USA. ${ }^{6}$ Department of Cell and Developmental Biology, Vanderbilt University, Nashville, Tennessee, USA. ${ }^{7}$ Department of Veterans Affairs Medical Center, Nashville, Tennessee, USA. ${ }^{8}$ Translational Genomics Research Institute, Phoenix, Arizona, USA. ${ }^{9}$ The Vanderbilt COVID-19 Consortium Cohort is detailed in the Supplemental Acknowledgments. ${ }^{10}$ The Human Cell Atlas Biological Network is detailed in the Supplemental Acknowledgments.

\begin{abstract}
The severe acute respiratory syndrome coronavirus 2 (SARS-CoV-2) novel coronavirus 2019 (COVID-19) global pandemic has led to millions of cases and hundreds of thousands of deaths. While older adults appear at high risk for severe disease, hospitalizations and deaths due to SARS-CoV-2 among children have been relatively rare. Integrating single-cell RNA sequencing (scRNA-seq) of developing mouse lung with temporally resolved immunofluorescence in mouse and human lung tissue, we found that expression of SARS-CoV-2 Spike protein primer TMPRSS2 was highest in ciliated cells and type I alveolar epithelial cells (AT1), and TMPRSS2 expression increased with aging in mice and humans. Analysis of autopsy tissue from fatal COVID-19 cases detected SARS-CoV-2 RNA most frequently in ciliated and secretory cells in airway epithelium and AT1 cells in peripheral lung. SARS-CoV-2 RNA was highly colocalized in cells expressing TMPRSS2. Together, these data demonstrate the cellular spectrum infected by SARS-CoV-2 in lung epithelium and suggest that developmental regulation of TMPRSS2 may underlie the relative protection of infants and children from severe respiratory illness.
\end{abstract}

\section{Introduction}

The emergence of the severe acute respiratory syndrome coronavirus 2 (SARS-CoV-2) novel coronavirus 2019 (COVID-19) has led to a global pandemic, with more than 35 million cases as of October 2020 (1). While the global burden of disease has overwhelmed healthcare systems around the world, severe illness and death from COVID-19 are rare in the pediatric population (2). One aspect of the COVID-19 pandemic that has eluded explanation is the striking diversity of clinical phenotypes accompanying SARS-CoV-2 infection, ranging from asymptomatic carriage to life-threatening multiorgan failure (3-6). Morbidity and mortality appear most severe among the elderly (7), while infection rates and hospitalizations among infants and children are substantially lower (1). Up to $90 \%$ of children infected with SARS-CoV-2 exhibit no or mild symptoms (2), and documented neonatal infections

Authorship note: JAK and JMSS contributed equally to this work.

Conflict of interest: JAK has received advisory board fees from Boehringer Ingelheim Inc. and has research contracts with Genentech. TSB has received advisory board fees from Boehringer Ingelheim Inc., Orinove, GRI Bio, Morphic, and Novelstar, and has research contracts with Genentech and Celgene.

Copyright: () 2021, American Society for Clinical Investigation

Submitted: June 1, 2020; Accepted: November 5, 2020; Published: November 12, 2020.

Reference information: J Clin Invest. 2021;131(1):e140766.

https://doi.org/10.1172/JCl140766. remain rare. This led us to hypothesize that host factors governing SARS-CoV-2 infectivity and attachment in the lower respiratory epithelium may be developmentally regulated. Differential expression of viral attachment and entry factors across lung development may provide biologic rationale for the variability in COVID-19 presentation.

\section{Results and Discussion}

To investigate how the expression of genes associated with SARSCoV-2 susceptibility changes during lung development, we analyzed a previously unpublished single-cell RNA sequencing (scRNAseq) data set profiling the epithelial and stromal cells in developing mouse lung at 5 time points ranging from embryonic day 18 (E18) to postnatal day 64 (P64) (Figure 1, A and B, Supplemental Figure $1, \mathrm{~A}$ and $\mathrm{B}$; supplemental material available online with this article; https://doi.org/10.1172/JCI140766DS1). We interrogated expression profiles of genes linked to SARS-CoV-2 infectivity by analyzing a total of 67,629 cells across these 5 time points (Figure 1, C and D). Previous work suggests that SARS-CoV-2 gains cellular entry by binding ACE2 on the cell surface (8-10) and fusing with the cell membrane after the spike-protein undergoes protease-mediated cleavage (11). TMPRSS2 is the canonical protease mediating cellular entry for coronaviruses, including SARS-CoV-2 (11), but other proteases have also been implicated in this process (10). Consistent with 
A

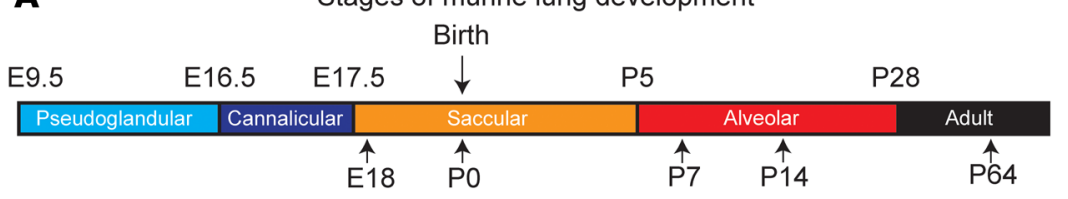

B

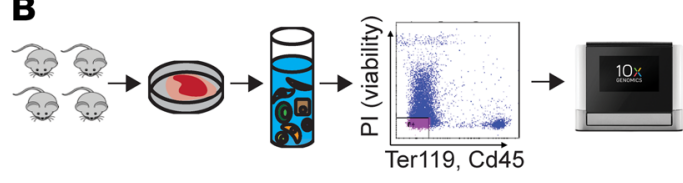

Timepoints for lung development scRNA-seq time series

C
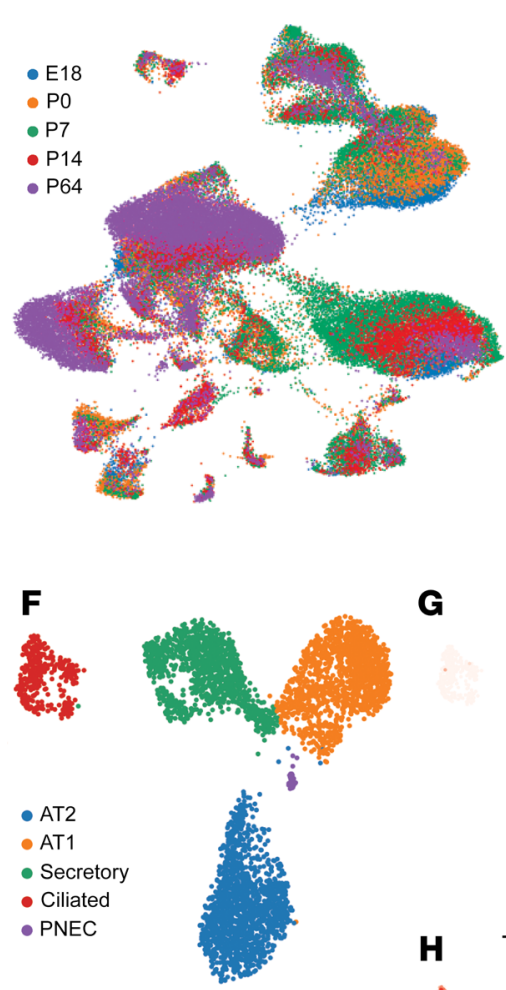

D

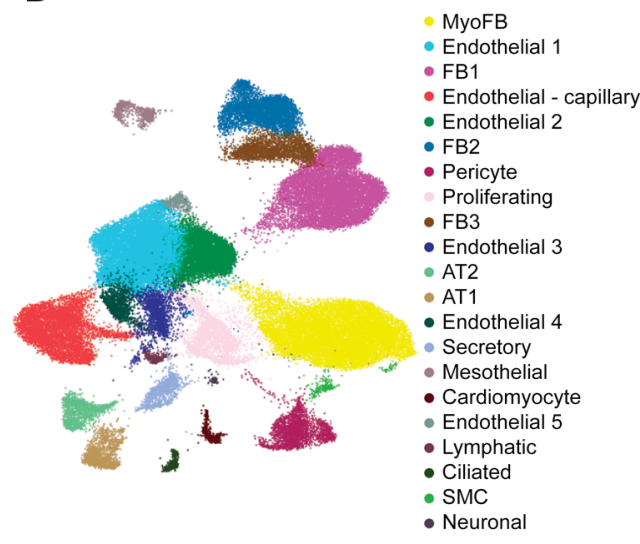

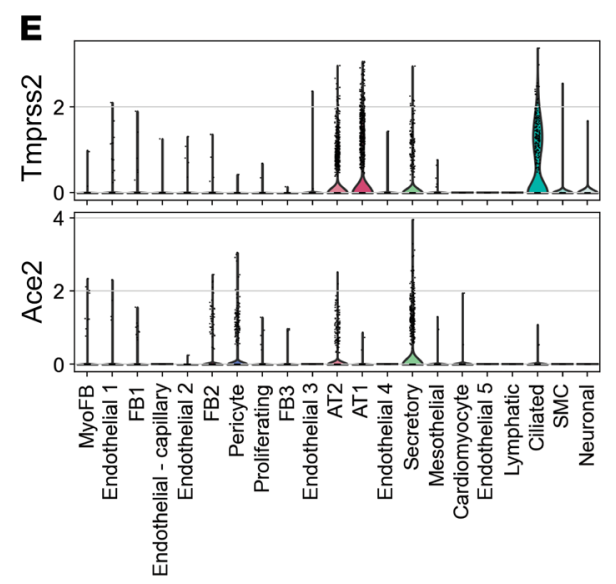

G

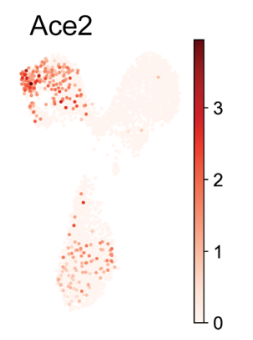

H Tmprss2

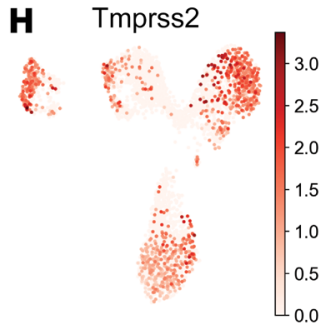

I Ace2
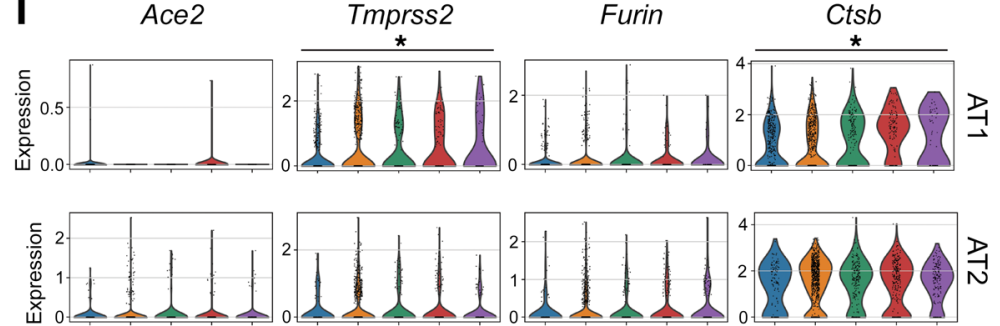

Figure 1. Time-series scRNA-seq of developing mouse lung. (A) Overview of murine developmental stages and time points for scRNA-seq time series. (B) Workflow of scRNA-seq time series. Single-cell suspensions were generated from at least 4 mice at each time point. Viable, Cd45-, Ter119- cells were selected for scRNA-seq library preparation using the 10X Cenomics Chromium 5' platform. Uniform Manifold Approximation and Projection (UMAP) embedding of 67,629 cells annotated by (C) developmental time point and (D) cell-type. (E) Violin plot depicting expression of key SARS-CoV2 receptor Ace 2 and coreceptor Tmprss 2 across cell types. (F) UMAP embedding of 4,369 epithelial cells after subsetting and reclustering. (G, H) UMAP plots depicting relative expression of Ace2 and Tmprss2. (I) Relative expression of putative SARS-CoV-2 priming proteases during development, shown as violin plots to indicate relative expression, with individual cells shown as dots. ${ }^{*} P<3.125 \times 10^{-3}$ by 1 -way ANOVA across developmental time points.

recent analysis of single-cell transcriptomic data of the lung and other organs (12-15), we observed that during lung development, expression of Ace2 was generally low, largely restricted to epithelial cells, most frequently and most highly expressed in secretory cells (Figure 1E), and expressed in a small subset of alveolar type 2 (AT2) cells. In contrast, Tmprss 2 was expressed broadly in the epithelium and most highly expressed in ciliated cells and alveolar type 1 (AT1) cells (Figure 1, E-I). A small proportion of fibroblasts and pericytes expressed Ace2, but there was minimal Ace 2 or Tmprss 2 in endo- thelial or other stromal cells (Figure 1E). Examining the relative expression of Tmprss 2 and other putative priming proteases across developmental time (including furin and cathepsin B), we observed that Tmprss 2 expression in ciliated cells was significantly higher at P64 compared with all earlier developmental time points; a similar pattern was observed for Ctsb. In AT1 cells, Tmprss2 expression generally increased during alveolarization into adulthood.

To spatially and temporally localize expression of Tmprss2, we performed RNA in situ hybridization (RNA-ISH) with automated 
A Scgb1a1/ Tmprss2
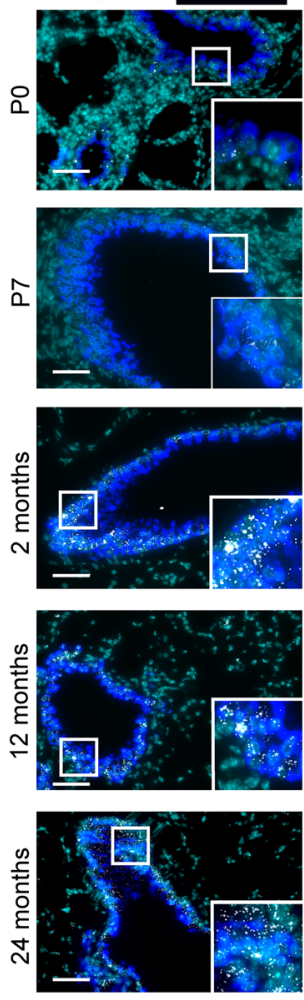

Secretory cells
Foxj1/Tmprss2
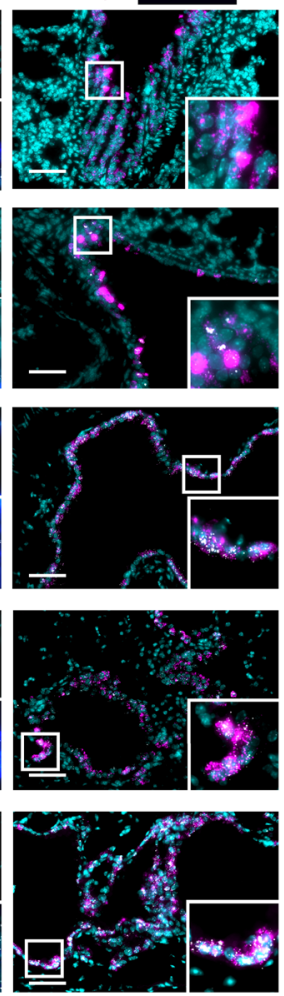

Ciliated cells
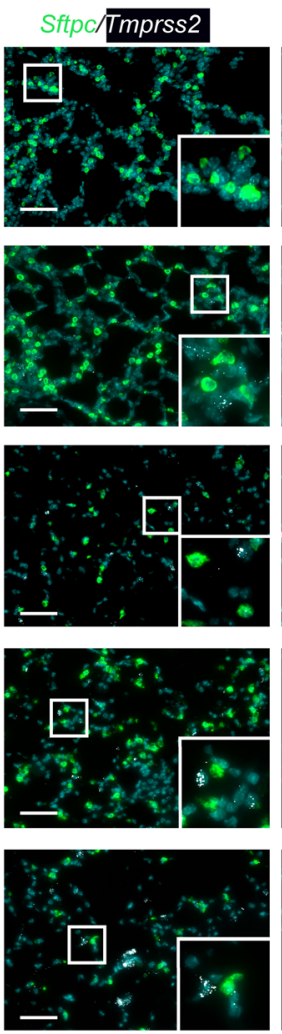

AT2 cells
Hopx/Tmprss2
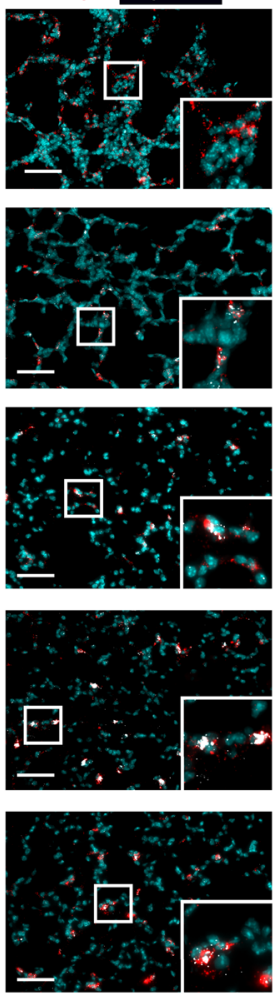

AT1 cells
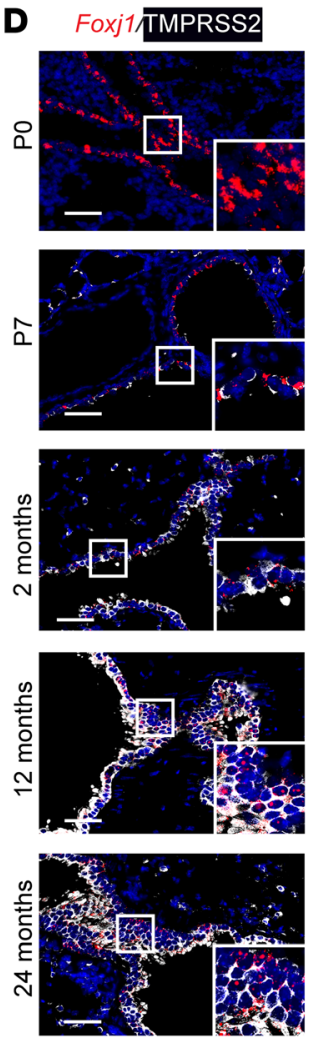

Hopx/TMPRSS2
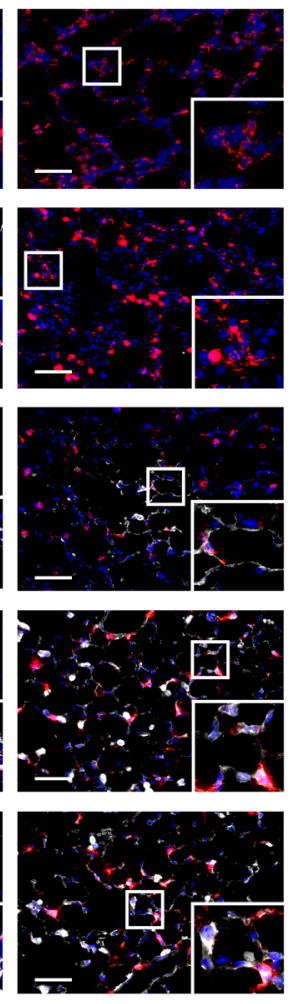

B

Tmprss2 expression in Scgb1a1+ cells
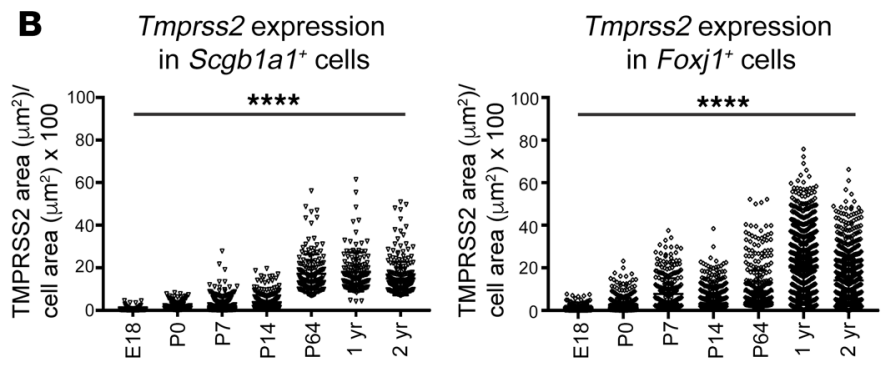

Tmprss2 expression in $\mathrm{Sftpc}^{+}$cells

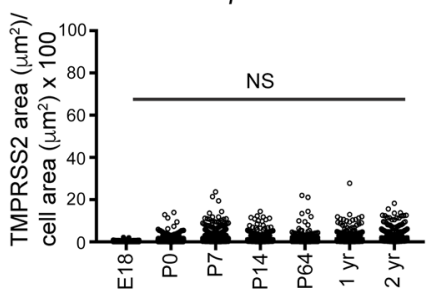

Tmprss2 expression in Hopx ${ }^{+}$cells

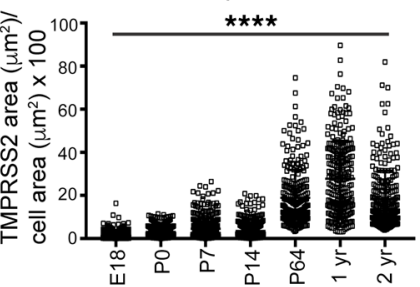

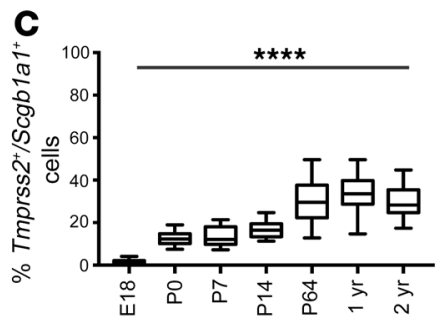
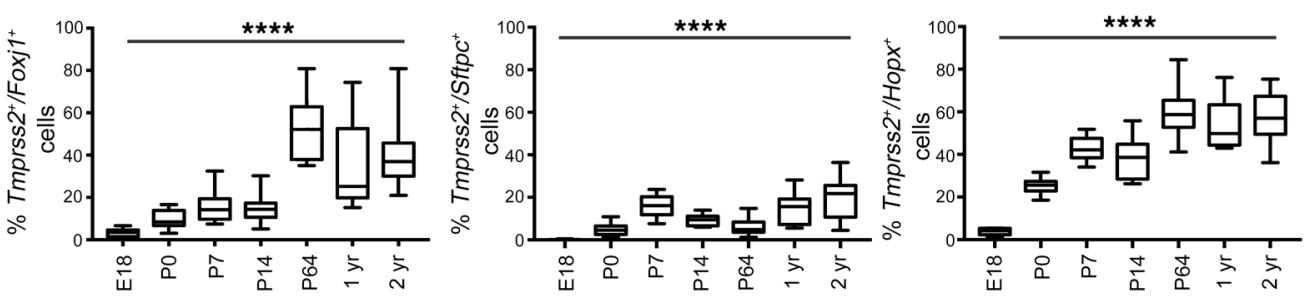

Figure 2. Spatial and temporal localization of Tmprss2 expression across lung development. (A) RNA-ISH of Tmprss2 expression (white) with epithelial markers Scgb1a1 (secretory cells, blue), Foxj1 (ciliated cells, magenta), Sftpc (surfactant protein C, AT2 cells, green), Hopx (type 1 alveolar epithelial cells, red). FFPE tissue from lungs at E18, P0, P7, P14, 2 months, 12 months, and 24 months was used, with representative image data from P0, P7, 2 months, 12 months, and 24 months shown in the figure. Lungs from 3 mice at each time point were used, with ten $\times 40$ images obtained per slide. Scale bars: $100 \mu \mathrm{m}$. (B, C) Quantification of Tmprss2 expression in each epithelial subtype across development by automated image analysis (HALO, Indica Labs) measured as (B) percentage of cellular area covered by Tmprss2 probe in each cell expressing both Tmprss2 and the epithelial marker (all data points are shown with mean \pm SD) and (C) percentage of cells expressing the epithelial marker that also express Tmprss2, with positive Tmprss 2 expression defined as having 5 or more copies of Tmprss 2 probe (box and whisker plots are shown with mean and error bars reflecting minimum and maximum values for each time point). More than 1000 cells were counted at each time point. ${ }^{* * *} P<0.0001$ by 1-way ANOVA. (D) RNA-ISH of Foxj1 expression (red) or Hopx expression (red) with protein immunofluorescence for TMPRSS2 protein (white). Five $\times 40$ images per slide were obtained. Scale bars: $100 \mu \mathrm{m}$. 
A SCGB1A1/TMPRSS2
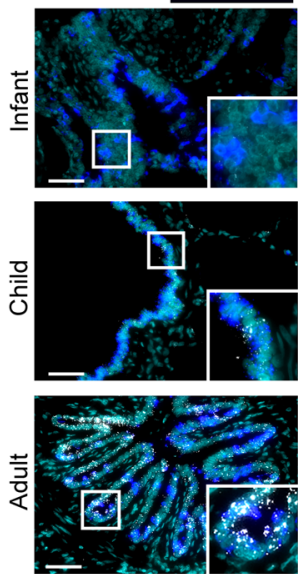

Secretory cells
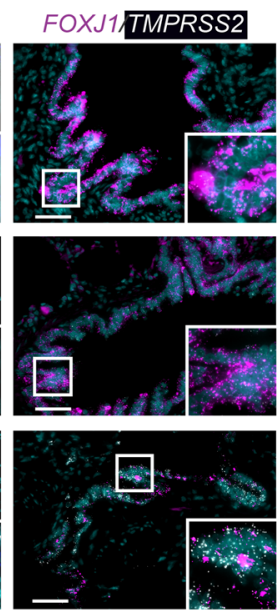

Ciliated cells
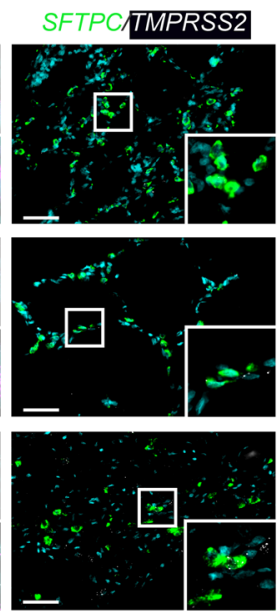

AT2 cells
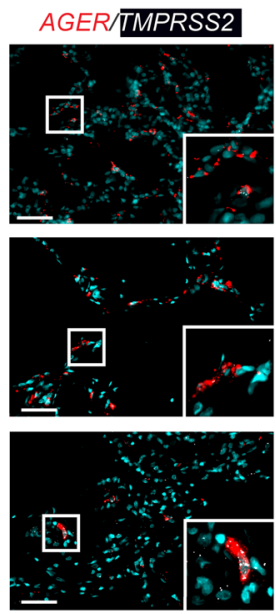

AT1 cells
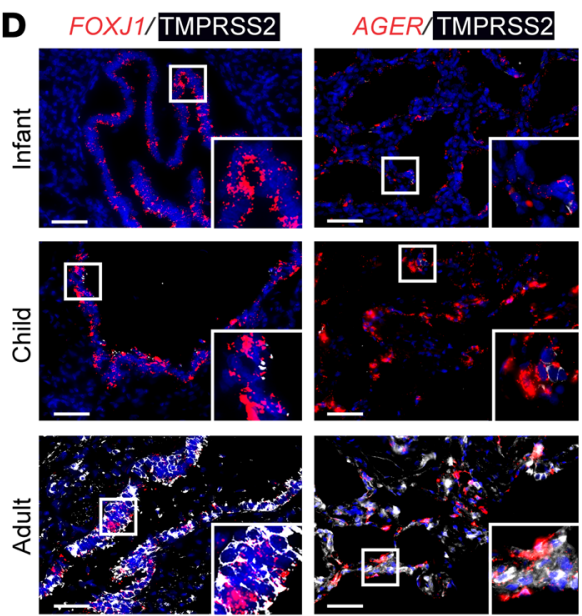

$\mathbf{B}$

TMPRSS2 expression in SCGB $1 A 1^{+}$cells
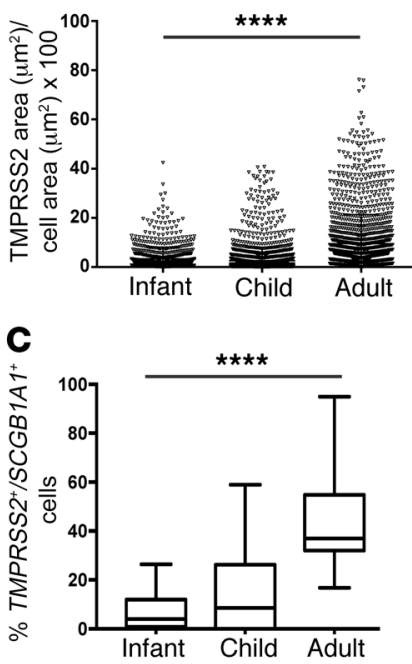

TMPRSS2 expression in $\mathrm{FOXJ1}^{+}$cells
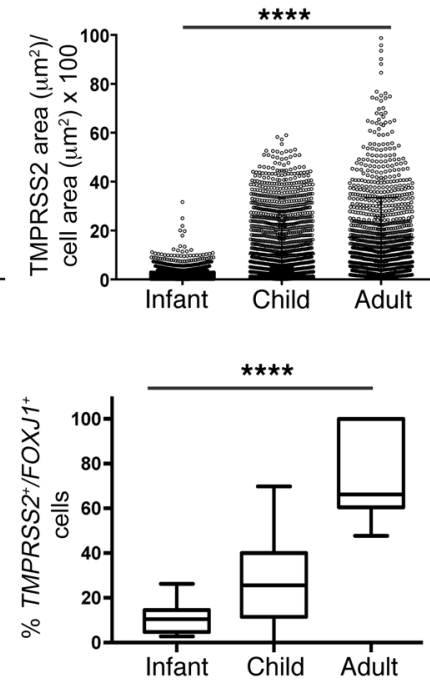

TMPRSS2 expression in SFTPC ${ }^{+}$cells
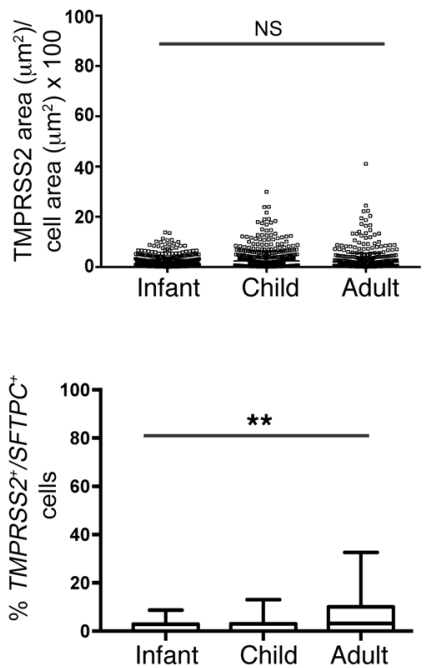

TMPRSS2 expression in $A G E R^{+}$cells

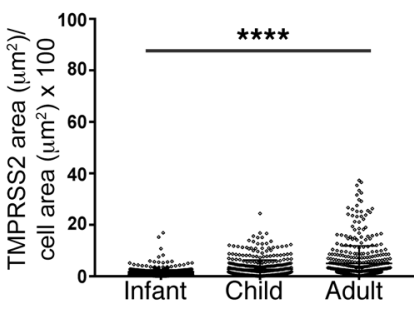

Figure 3. Spatial and temporal localization of TMPRSS2 expression in lung across the human lifespan. (A) RNA-ISH of TMPRSS2 expression (white) with epithelial markers SCGB1A1 (secretory cells, blue), FOXJ1 (ciliated cells, magenta), SFTPC (surfactant protein C, AT2 cells, green), AGER (AT1 cells, red). FFPE tissue from 20 human lungs between the ages of birth and 69 years was analyzed. Ten $\times 40$ images were obtained per slide for analysis. Scale bars: 100 $\mu \mathrm{m}$. (B, C) Quantification of TMPRSS2 expression in each epithelial subtype across development by automated image analysis (HALO, Indica Labs), measured as (B) percentage of cellular area covered by TMPRSS2 probe for each cell expressing both TMPRSS2 and the epithelial marker (all data points are shown with mean \pm SD and (C) percentage of cells expressing the epithelial marker that also express TMPRSS2, with positive TMPRSS2 expression defined has having 5 or more copies of TMPRSS2 probe (box and whisker plots are shown with mean and error bars reflecting minimum and maximum values for each time point). More than 1000 cells were counted at each time point. ${ }^{* * *} P<0.0001,{ }^{* *} P<0.01$ by 1 -way ANOVA. (D) RNA-ISH of FOXJ1 expression (red) or AGER expression (red) with protein immunofluorescence for TMPRSS2 protein (white). Data are shown with mean \pm SD. Five $\times 40$ images per slide were obtained. Scale bars: $100 \mu \mathrm{m}$.

quantification (HALO, Indica Labs) on time points corresponding to our scRNAseq (with the addition of adult and aged mice) using formalin-fixed paraffin-embedded (FFPE) lung tissue. Colocalizing Tmprss2 with Scgb1a1 (secretory cells), Foxj1 (ciliated cells), Sftpc (AT2 cells), and Hopx (AT1 cells) (Figure 2A), we observed an age-dependent progressive increase in the proportion of Tmprss $2^{+}$cells (Figure 2, B and C). Further, in AT1 cells and ciliated cells, there was a marked increase in relative Tmprss 2 expression across developmental time, which was most striking at 1 and 2 years of age at both the RNA and protein levels (Figure 2, B and D). Tmprss 2 expression was low in secretory cells, although levels increased in adult and aged mice (Figure 2, A-D). AT2 cell expression of Tmprss 2 was lower than other epithelial cells, and did not significantly increase across lung development. Notably, there was minimal detection of Tmprss 2 prenatally at E18, and relatively low levels in the saccular stage at PO in all epithelial cell types. Combining RNA-ISH with immunofluorescence for TMPRSS2 protein showed concordance between expression of the Tmprss 2 gene and TMPRSS2 protein (Supplemental Figure 2). The localization of TMPRSS2 protein expression in ciliated cells expressing Foxj1 and in AT1 cells expressing Hopx was also confirmed (Figure 2D). Consistent with our transcriptomic data, basal Ace2 expres- 
A
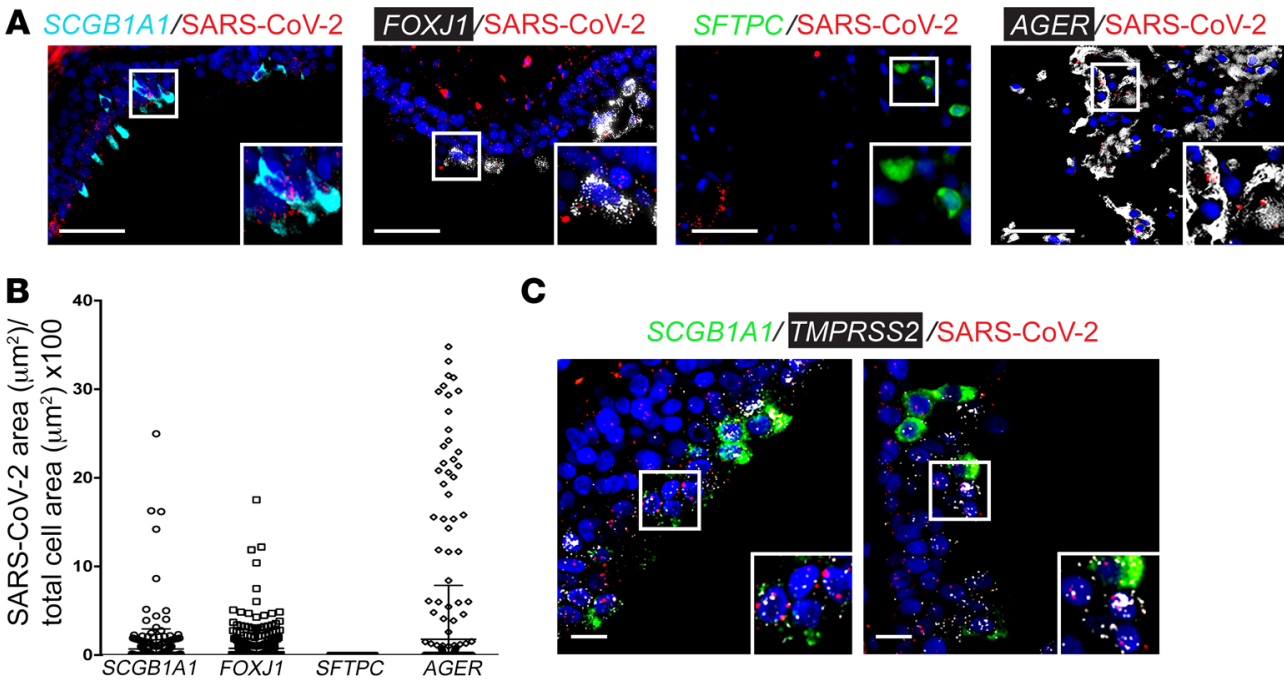

C

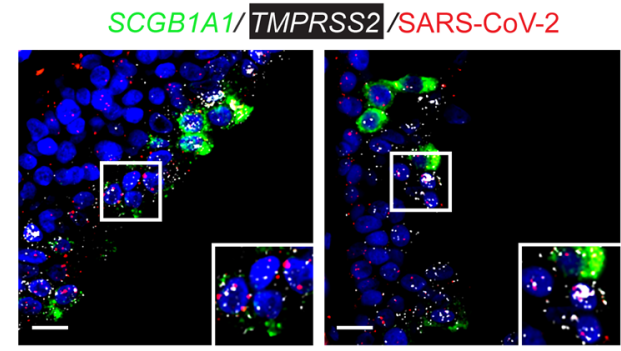

D

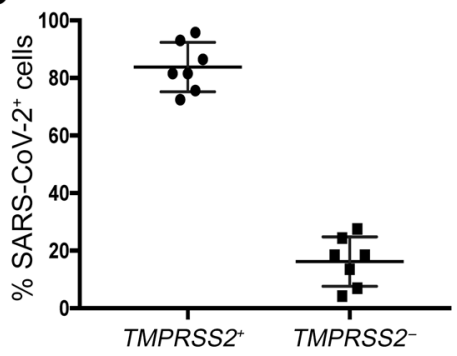

E

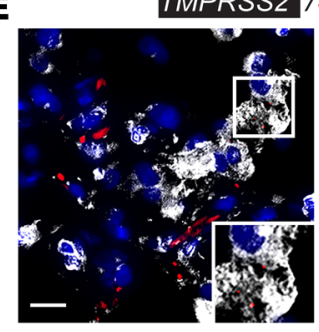

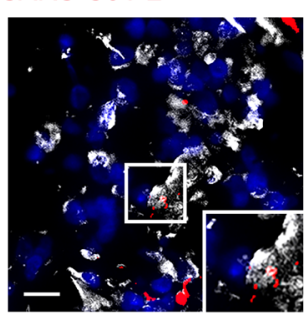

Figure 4. Spatial localization of SARS-CoV-2 RNA in lung autopsy tissue from fatal COVID-19. (A) RNAISH of SARS-CoV-2 RNA (red) with epithelial markers SCCB1A1 (secretory cells, cyan), FOXJ1 (ciliated cells, white), SFTPC (AT2 cells, green), AGER (AT1 cells, white). Scale bars: $100 \mu \mathrm{m}$. (B) Quantification of cells containing SARS-CoV-2 RNA by epithelial subtype as determined by percentage of cellular area covered by SARSCoV- 2 probe in all cells positive for the epithelial marker; more than 150 cells were counted for each subtype. All data points are shown with mean \pm SD. (c) RNA-ISH of large airway from the same patient demonstrating RNA transcripts for TMPRSS2 (white) in the same cells containing SARS-CoV-2 RNA (red), with secretory cells labeled in green (SCGB1A1) for context. Scale bars: $100 \mu \mathrm{m}$. (D) Quantification of TMPRSS2 expression in SARS-CoV-2+ cells; more than 1000 cells were counted from the 3 subjects, $P<0.05$ All percentages are shown in graphs with mean \pm SD. (E) RNA-ISH of SARS-CoV-2 (red) with protein immunofluorescence for TMPRSS2 protein (white). Scale bars: $100 \mu \mathrm{m}$. sion detected by RNA-ISH was low with little change during development and aging (Supplemental Figure 3).

To determine whether TMPRSS2 expression increases with aging in humans, we examined TMPRSS 2 expression by RNA-ISH with automated quantification and by immunofluorescence using a monoclonal antibody to TMPRSS2 in lung tissue from samples across the human lifespan. We defined infants as individuals up to 2 years of age $(n=7)$ and children as individuals between the ages of 3 and $17(n=9)$. Adult specimens were from subjects aged 54-69 (never smokers, $n=4$ ). Infants expressed TMPRSS2 at very low levels across all 4 epithelial lineages evaluated, whereas children exhibited similarly low levels of TMPRSS2 in secretory and alveolar epithelial cells with a significant increase in $\mathrm{FOXJ1}^{+}$ciliated cells (Figure 3, A-C). Adult subjects had higher TMPRSS2 expression in secretory, ciliated, and AT1 cells relative to both pediatric groups with very little TMPRSS 2 expression in AT2 cells (Figure 3, B and C). Similar to what we observed in mice, combining RNA-ISH with immunofluorescence for TMPRSS2 protein showed a high degree of concordance between Tmprss 2 gene expression and TMPRSS2 protein (Supplemental Figure 4). The localization of TMPRSS2 protein in ciliated cells expressing FOXJ1 and in AT1 cells expressing AGER, and the substantial increase of TMPRSS2 protein expression with aging, was consistent with RNA-ISH findings (Figure 3D). Human lung tissue demonstrated low levels of ACE2 expression in infants, children, and adult samples (Supplemental Figure 5). These data are broadly consistent with the results of a single-nucleus RNA-seq (snRNA-seq) study of infants, children, and adults up to age 30 that was reported while this manuscript was being finalized, though notably that study did not show tissue validation of their findings (16).
To understand the relevance of our findings in the setting of COVID-19 infection, we analyzed autopsy specimens from the lungs of 3 patients who died from complications of COVID-19. Using RNA-ISH with automated quantification, we identified the presence of SARS-CoV-2 RNA in both large airways and lung parenchyma. SARS-CoV-2 localized to epithelial cells expressing SCGB1A1 (secretory), FOXJ1 (ciliated), and AGER (AT1) (Figure 4, A and B, Supplemental Figure 8, Supplemental Figure 9). Surprisingly, despite analyzing more than $150 \mathrm{SFTPC}^{+}$cells (corresponding to AT2), none contained detectable SARS-CoV-2 RNA by this assay (Figure 4, A and B). In large airways (mainstem bronchus), HALO analysis showed that SARS-CoV-2 colocalized with TMPRSS2 in SCGB1A1 ${ }^{+}$secretory cells as well as SCGB1A1(presumably ciliated) cells (Figure 4C). Across autopsy specimens, SARS-CoV-2 RNA was highly colocalized in cells expressing TMPRSS2 (Figure 4D). Since autopsy tissue likely represents end-stage disease, the surprising absence of SARS-CoV-2 in AT2 cells could be explained by changes in viral replication during the course of the illness and/or increased cytotoxicity of infected AT2 cells; alternatively, direct viral infection of AT2 cells may not play as significant a role in disease pathophysiology as hypothesized. It is also possible that SARS-CoV-2 localizes to other cells in the lung, such as inflammatory cells. These data indicate that secretory, ciliated, and AT1 cells had the highest levels of TMPRSS2 expression and that these cell types all harbored SARS-CoV-2 RNA in lung tissue from severely infected patients, similar to data observed in an ex vivo infection model (17). Immunofluorescence for TMPRSS2 protein showed localization of SARS-CoV-2 virus in cells expressing TMPRSS2 (Figure 4E). Together, these data suggest that prim- 
ing protease TMPRSS2 expression may be an important determinant of SARS-CoV-2 infectivity in the lower respiratory tract.

The epithelial cell tropism of SARS-CoV-2 is dependent upon viral attachment and activation by cell-surface proteins, e.g., ACE2 and TMPRSS2. While ACE2 has been well studied in in vitro platforms of differentiated lung epithelium, our findings and prior studies $(12-14,18)$ suggest that ACE2 is expressed at low levels in respiratory epithelium (Figure 1, Supplemental Figure 3, Supplemental Figure 5). Conversely, rising expression of TMPRSS2 with age in both conducting airways and alveolar epithelium may explain the increased susceptibility to symptomatic infection and severe ARDS observed in adults relative to children. To date, there have been very few cases of vertical transmission of SARS-CoV-2 to newborns (19), despite viral particles being identified in the placentas of women infected with SARS-CoV-2 (20).

In light of the very low expression of $A C E 2$ in ciliated epithelial cells, it is possible that ACE2-independent attachment may play a role in SARS-CoV-2 cellular entry and pathogenesis. CD147 (basigin $[B S G])$ has been proposed as an alternative SARS-CoV-2 receptor (21) and recent work has examined the expression of $B S G$ and other putative SARS-CoV-2 receptors in adult respiratory epithelium (22). In our scRNAseq data, Bsg expression increased in ciliated cells with development and decreased with age in other epithelial cell types (Supplemental Figure 6, A and B). Alternatively, recent data indicate that ACE2 may be induced by interferon signaling, with viral entry propagating ACE2 expression in neighboring cells by this mechanism (13). In our scRNAseq time series, we observed an increasing interferon signature with age in lung epithelial cells, most prominent in AT2 and AT1 cells (Supplemental Figure 7, A and B). This raises the possibility that the developing lung may have relatively less interferon priming and therefore have less efficient ACE2 induction after viral infection. We did not observe developmental differences in basal expression of innate inflammatory genes linked to coronaviruses response, including Irf3, Tnf, Mapk1/3, Nfkb1/2, and Cxcl15 (Supplemental Figure 7C) (13, 23-25).

The very low levels of TMPRSS 2 expression in human infants, and nearly absent levels of Tmprss 2 expression in prenatal mice, suggest a mechanism by which neonates may be relatively protected against severe forms of COVID-19. After the SARS outbreak in 2003, several groups (26-28) reported that proteolytic cleavage of the SARS-CoV spike protein is a prerequisite for viral activation and host cell entry, with SARS-CoV tropism for TMPRSS2expressing cells in primates (29). Inhibition of TMPRSS2 with the serine protease inhibitor Camostat, partially prevented SARS-CoV infection (30), and addition of a cathepsin inhibitor (Aloxistatin) to Camostat potentiated the antiviral effect (30).These data highlight the potential for TMPRSS2 inhibition as an effective strategy for reducing SARS-CoV-2 infection, although additional animal studies are needed to understand whether TMPRSS2 is required for SARS-CoV-2 entry in vivo.

In summary, these data suggest that developmental regulation of viral entry factors may be the primary determinant of age- related differences in SARS-CoV-2 susceptibility and severity. The identification of changes in TMPRSS 2 expression associated with age presents a biological rationale for the observed rarity of severe lower respiratory tract SARS-CoV-2 disease in children, and underscores the opportunity to consider TMPRSS2 inhibition as a potential therapeutic target for SARS-CoV-2.

\section{Methods}

Methods are described in detail in the supplemental material.

Data availability. Raw and processed data used in generating this data set are available in the Gene Expression Omnibus (GEO) at NCBI with accession number GSE160876. Count matrices are available for download as Supplemental Table 1. Code used for data set integration and analyses in this manuscript is available at https:// github.com/KropskiLab.

Study approval. For the use of human tissue for research, all studies were approved by the Vanderbilt Institutional Review Board (nos. 060165, 171657, 200490). Relatives consented for autopsy tissue to be used for research. The protocol for animal research was approved by the Institutional Animal Care and Use Committee of Vanderbilt University and was in compliance with the Public Health Services policy on humane care and use of laboratory animals.

\section{Author contributions}

JMSS and JAK conceived the study. EJP, JTB, CJT, CJ, PG, DSN, $\mathrm{NMN}$, and $\mathrm{AH}$ performed experiments. BAS, ACH, NEB, JMSS, and JAK analyzed transcriptomic data. MK, SHG, and TSB provided samples. BAS, ACH, JAK, MEK, and JMSS interpreted data with assistance from TSB, SHG, SAW, NEB, LZB, and MK. BAS, LZB, JAK and JMSS wrote the manuscript and all authors provided critical feedback on the manuscript.

\section{Acknowledgments}

This work was supported by NIH grants K08HL143051 (to JMS), K08HL130595 (to JAK), R01HL145372 (to JAK/NEB), P01HL092470 (to TSB), K08HL127102 (to EJP), K08HL133484 (to JTB), R01AI077505 (to DWH), P30AI110527 (to SAM), R01AI142095 (to SAK/SAM), and TR002243. JAK and JMSS were funded by the Francis Family Foundation. Flow cytometry experiments were performed in the VMC Flow Cytometry Shared Resource, which is supported by the Vanderbilt Ingram Cancer Center (P30 CA68485) and the Vanderbilt Digestive Disease Research Center (DK058404). See Supplemental Acknowledgments for details on the Vanderbilt COVID-19 Consortium Cohort and the Human Cell Atlas Biological Network.

Address correspondence to: Jennifer M.S. Sucre, 2215 Garland Avenue, 1125 Light Hall, Nashville, Tennessee 37232, USA. Phone: 615.343.0826; Email: jennifer.sucre@vumc.org. Or to: Jonathan A. Kropski, 1161 21st Avenue S, MCN, Suite T-1218, Nashville, Tennessee 37232, USA. Phone: 615.875.7935; Email: jon.kropski@ vumc.org.

\footnotetext{
1. Johns Hopkins Coronavirus Resource Center. https://coronavirus.jhu.edu/. Accessed November 10, 2020.

2. Dong Y, et al. Epidemiology of COVID-19
}

among children in China. Pediatrics. 2020;145(6):e20200702.

3. Grasselli G, et al. Baseline characteristics and outcomes of 1591 patients infected with SARS-
CoV-2 admitted to ICUs of the Lombardy region, Italy. JAMA. 2020;323(16):1574-1581.

4. Richardson S, et al. Presenting characteristics, comorbidities, and outcomes among 5700 
patients hospitalized with COVID-19 in the New York City area. JAMA. 2020;323(20):2052-2059.

5. Guan WJ, et al. Clinical characteristics of coronavirus disease 2019 in China. N Engl J Med. 2020;382(18):1708-1720.

6. Bai Y, et al. Presumed asymptomatic carrier transmission of COVID-19. JAMA. 2020;323(14):1406-1407.

7. Wu C, et al. Risk factors associated with acute respiratory distress syndrome and death in patients with coronavirus disease 2019 pneumonia in Wuhan, China. JAMA Intern Med. 2020;180(7):934-943.

8. Letko M, Marzi A, Munster V. Functional assessment of cell entry and receptor usage for SARS$\mathrm{CoV}-2$ and other lineage $\mathrm{B}$ betacoronaviruses. Nat Microbiol. 2020;5(4):562-569.

9. Luan J, et al. SARS-CoV-2 spike protein favors ACE2 from Bovidae and Cricetidae. J Med Virol. 2020;92(9):1649-1656.

10. Walls AC, Park YJ, Tortorici MA, Wall A, McGuire AT, Veesler D. Structure, function, and antigenicity of the SARS-CoV-2 spike glycoprotein. Cell. 2020;181(2):281-292.e6.

11. Hoffmann M, et al. SARS-CoV-2 cell entry depends on ACE2 and TMPRSS2 and is blocked by a clinically proven protease inhibitor. Cell. 2020;181(2):271-280.e8.

12. Sungnak $\mathrm{W}$, et al. SARS-CoV-2 entry factors are highly expressed in nasal epithelial cells together with innate immune genes. Nat Med. 2020;26(5):681-687.

13. Ziegler CGK, et al. SARS-CoV-2 receptor ACE2 is an interferon-stimulated gene in human airway epithelial cells and is detected in specific cell subsets across tissues. Cell. 2020;181(5):1016-1035.e19.

14. Muus C LM, et al. Integrated analyses of singlecell atlases reveal age, gender, and smoking status associations with cell type-specific expression of mediators of SARS-CoV-2 viral entry and high- lights inflammatory programs in putative target cells. bioRxiv 2020;2020.04.19.049254. https:// doi.org/10.1101/2020.04.12.037580. Published online April 14, 2020. Accessed November 10, 2020.

15. Zou X, Chen K, Zou J, Han P, Hao J, Han Z. Single-cell RNA-seq data analysis on the receptor ACE2 expression reveals the potential risk of different human organs vulnerable to $2019-\mathrm{nCoV}$ infection. Front Med. 2020;14(2):185-192.

16. Wang A CJ, Poirion O, Buchanan J, Valdez M, Verheyden J, et al. Single nucleus multiomic profiling reveals age-dynamic regulation of host genes associated with SARS-CoV-2 Infection. bioRxiv. 2020;2020.04.12.037580. https://doi. org/10.1101/2020.04.12.037580. Published online April 14, 2020. Accessed online November 10, 2020.

17. Hui KPY, et al. Tropism, replication competence, and innate immune responses of the coronavirus SARS-CoV-2 in human respiratory tract and conjunctiva: an analysis in ex-vivo and in-vitro cultures. Lancet Respir Med. 2020;8(7):687-695.

18. Hikmet F, Méar L, Edvinsson Å, Micke P, Uhlén $\mathrm{M}$, Lindskog $\mathrm{C}$. The protein expression profile of ACE2 in human tissues. Mol Syst Biol. 2020;16(7):e9610.

19. Chen $\mathrm{H}$, et al. Clinical characteristics and intrauterine vertical transmission potential of COVID-19 infection in nine pregnant women: a retrospective review of medical records. Lancet. 2020;395(10226):809-815.

20. Algarroba GN, et al. Visualization of severe acute respiratory syndrome coronavirus 2 invading the human placenta using electron microscopy. $A m \mathrm{~J}$ Obstet Gynecol. 2020;223(2):275-278.

21. Wang K, et al. SARS-CoV-2 invades host cells via a novel route: $\mathrm{CD} 147$-spike protein. bioRxiv.2020.03.14.988345. https://doi. org/10.1101/2020.03.14.988345. Published March 14, 2020. Accessed online November 10, 2020.
22. Aguiar JA, et al. Gene expression and in situ protein profiling of candidate SARS-CoV-2 receptors in human airway epithelial cells and lung tissue. Eur Respir J. 2020;56(3):2001123.

23. Huang IC, et al. Distinct patterns of IFITMmediated restriction of filoviruses, SARS coronavirus, and influenza A virus. PLoS Pathog. 2011;7(1):e1001258.

24. Fung TS, Liu DX. Human coronavirus: host-pathogen interaction. Annu Rev Microbiol. 2019;73:529-557.

25. Zhao X, et al. Interferon induction of IFITM proteins promotes infection by human coronavirus OC43. Proc Natl Acad Sci US A. 2014;111(18):6756-6761.

26. Simmons G, Reeves JD, Rennekamp AJ, Amberg SM, Piefer AJ, Bates P. Characterization of severe acute respiratory syndrome-associated coronavirus (SARS-CoV) spike glycoproteinmediated viral entry. Proc Natl Acad Sci U S A. 2004;101(12):4240-4245.

27. Matsuyama S, Ujike M, Morikawa S, Tashiro M, Taguchi F. Protease-mediated enhancement of severe acute respiratory syndrome coronavirus infection. Proc Natl Acad Sci U S A. 2005;102(35):12543-12547.

28. Huang IC, et al. SARS coronavirus, but not human coronavirus NL63, utilizes cathepsin L to infect ACE2-expressing cells. J Biol Chem. 2006;281(6):3198-3203.

29. Matsuyama S, Nagata N, Shirato K, Kawase M, Takeda M, Taguchi F. Efficient activation of the severe acute respiratory syndrome coronavirus spike protein by the transmembrane protease TMPRSS2.J Virol. 2010;84(24):12658-12664.

30. Kawase M, Shirato K, van der Hoek L, Taguchi F, Matsuyama S. Simultaneous treatment of human bronchial epithelial cells with serine and cysteine protease inhibitors prevents severe acute respiratory syndrome coronavirus entry. J Virol. 2012;86(12):6537-6545. 